\title{
Simulation of Signals with Field Signal Simulator
}

\author{
${ }^{1}$ Pattan Munni, ${ }^{2}$ V.Vittal Reddy, ${ }^{3}$ N. Nageswararao \\ ${ }^{1}$ Student, ${ }^{2}$ Associate Professor, ${ }^{3}$ Assistant general manager \\ 1. Gudlavalleru Engineering College, 2.Gudlavalleru Engineering College, 3.ECIL Hydrabad Gudlavalleru, \\ Krishna District, Andhra Pradesh, India, Pin no.
}

\begin{abstract}
In the recent trends the field signal processing is an emerging technology for data acquisition systems, controlling application systems and automation system in real time environment. Versa Modular European (VME) and CRIO based hardware to simulate the field signals for the computer based control and instrumentation panel. Simulator is used to transmit multiple number of signals at a time. FSS software is a generic software to simulate the field signals for a computer based control and instrumentation system. Its general purpose nature easily extends its capabilities to build and perform unit under test(UUT's) ATP specific test routines. Field signal simulator (FSS)is also an Automatic Testing Equipment (ATE). The main the Scope of this project covers real time computer (RTC) systems used for Signal Processing \& Control application and the simulation techniques used to achieve automation by testing these RTC systems. It also includes RTC hardware and the software used for process \& control applications. Simulation hardware \& software used to test VME system is also included in the scope of this study.
\end{abstract}

\section{Introduction}

Real time computer system consisting of dual redundant VME bus based systems with Switch over Logic Systems; Test software thoroughly tests the systems to all the channels of each card present in VME bins.

Software also checks the healthiness of each card at every scan interval and sends the information to display station. The signal processing electronic system consists of ED20 CPU card and combination of I/O cards to meet the system reliability, functionality and to analyze the performance. To verify the system functionality and performance we need to test the system thoroughly. To simulate this system with any type of signal we need a field signal simulator to reduce processing time. Without this simulator testing is tedious process.

The existing simulator used as a passive simulator with switches for simulating digital inputs and potentiometers for analog inputs. For generating digital outputs LEDs were provided and DPMs for analog outputs. Using passive switch it was a very tedious process to test the RTC $[1,2]$ systems for all input conditions \& time consuming.

It introduces simulation techniques to simulate VME RTC systems with field signal simulator. FSS can simulate any type of input signal like $0.5 \mathrm{v}$ to $9.5 \mathrm{v} \mathrm{dc}, 4-20 \mathrm{~mA}$. It also implements simulation software for automating the testing process. VME systems software has to scan all the analog and digital input signals, receive the soft inputs/commands from process computer. It generates outputs for monitoring on display station and process computer.

In this paper describes RTC systems used for signal processing \& control application and the simulation techniques used to achieve automation by testing these RTC systems. It also includes RTC hardware and software used to test VME [3] system. RTC systems are deployed to check plant systems conditions and generate authorization outputs when all the conditions are satisfied. More than $1500 \mathrm{I} / \mathrm{O}$ signals can simulate at a time by Field Signal Simulator according to the system capability.

\section{A. Field Signal Simulator}

\section{System Design Model}

The Computer based Control System acquires various types of physical parameters, such as temperature, pressure, flow, strain, position, and speed using electronic equipment/ modules/boards. The data collected is sent to a computer for analysis and display. The control systems also take action (control) based on the data they receive. Turning ON/OFF lamps, motors, valves, heaters and fans are common functions of control. Sending out voltage, current, digital words, pulses and waveforms are also functions of the Control systems. Embedded software running in the computer coordinates and executes these functions. 


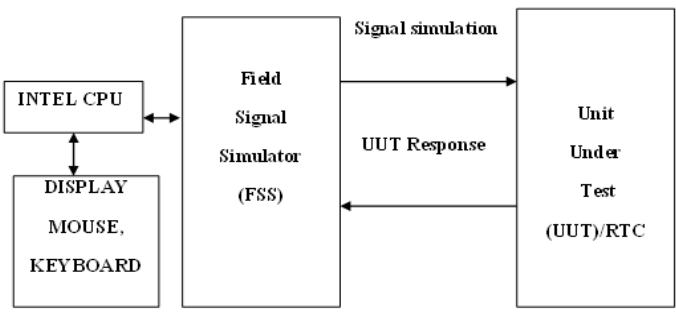

Fig: 1. Block diagram of Unit under Test with Field signal simulator

System architecture: The FSS is housed in standard racks with 19" card frames. The system operates on single-phase $230 \mathrm{~V}, 50 \mathrm{~Hz}, \mathrm{AC}$ power supply. The instrumentation is PXI and VME based, with an Industrial computer for simulation of field signals. High speed Ethernet link connects Industrial computer and the CPU module on the PXI bus. The system is designed to work up to 55 degrees Celsius. Battery back-up is provided for conducting the burn-in tests. Video graphic display, keyboard, mouse and color printer are connected to the PXI bus based INTEL CPU board as shown Figure 3.2.

Data Acquisition and Control: The MS Window operating system runs on the Intel Pentium CPU, on which the application software is developed using National Instrument's LABVIEW. The application software generates the field data for the signal conditioning boards using the hardware modules. The response parameters of the unit under test are read by the CPU. The FSS generates the test reports based on the Accepted Test Procedure of the Unit Under Test (UUT).

I/O Connections: FSS inputs and outputs are terminated on terminal blocks internally. All the cables are color coded and labeled for easy identification. A separate rack is provided for storing the cable bunches, whenever the system is not in use. All inputs and outputs are available simultaneously, no multiplexing of the signals as shown Figure 2.

Test Reports: FSS generates the test reports for the Project Engineers, Quality Control and Customer based on the Accepted Test Procedure

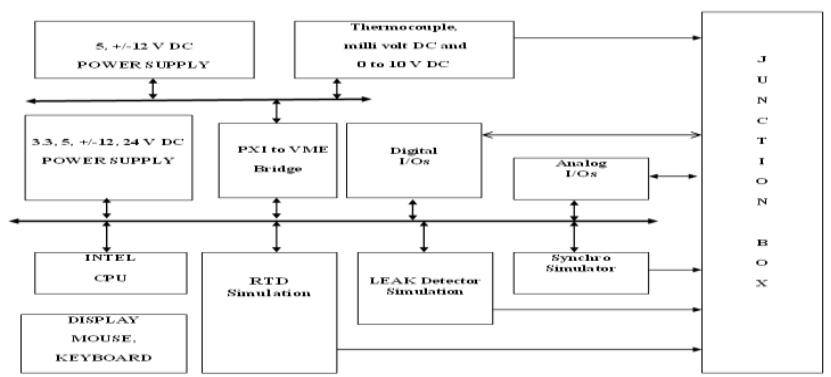

Fig: 2.Block diagram of I/O connection with Field signal simulator

\section{B. FSS Software}

FSS software is generic software to simulate that field signals for a computer based control and instrumentation system. FSS software works in conjunction with PXI, VME,CRIObased hardware to simulate the field signals for the computers based control and instrumentation panel. Its general purpose nature easily extends its capabilities to build and perform specific UUT's test routines.

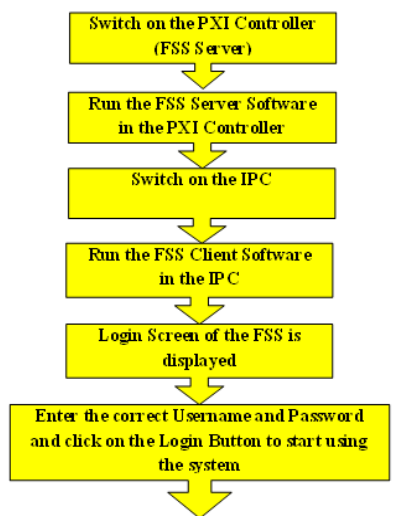

Fig: 3. SwITCH on Procedure for Server \& Client 
The FSS software is built following a "Client-Server" model. The FSS system consists of a PXI controller and two IPCs. PXI controller serves primarily as the Server and IPCs as Clients. One of the IPC will be designated as the "Development Station". Either the PXI-controller or the IPCs can be used as "Development station. It's highly recommended that PXI-Controller should not be used as a development station while its running the server $\mathrm{s} / \mathrm{w}$. Likewise IPCs should not be used as development station when its running the client $\mathrm{s} / \mathrm{w}$. The server $\mathrm{s} / \mathrm{w}$ in the PXI-controller runs on the background when it is launched. It should be launched before any client $\mathrm{s} / \mathrm{w}$ is launched. The PXI-Controller can also acts as a client in addition to being the server.

Thus, we can run the client $\mathrm{s} / \mathrm{w}$ on the PXI-controller itself which puts the usage of two IPCs as additional clients, only on need basis as shown Fig 3.

The following are the major modules of the Server $\mathrm{s} / \mathrm{w}$ are:

i) DAQCore: This module is the heart of the FSS software. DAQCore implements hardware level interaction with the PXI, VME and cRIO which constitutes the FSS systems. It calls the APIs built for the FSS systems and executes the commands sent by the Clients. DAQCore is a single module through which all interaction with all different types of hardware used in the FSS system is possible. DAQCore implements a common $\mathrm{I} / \mathrm{O}$ read/write errors handling mechanism and it also returns the value that are set through the commands.

ii) Command Router: Server hosts the command router module. Command Router module is used for identification of the origin of command for the DAQCore to execute. After the command is executed by the DAQCore the response is again routed through the Command Router to the source, the command router module also sorts the commands from various sources and segregates them based on the signal type for the DAQCore to execute. The source of the command could be any.

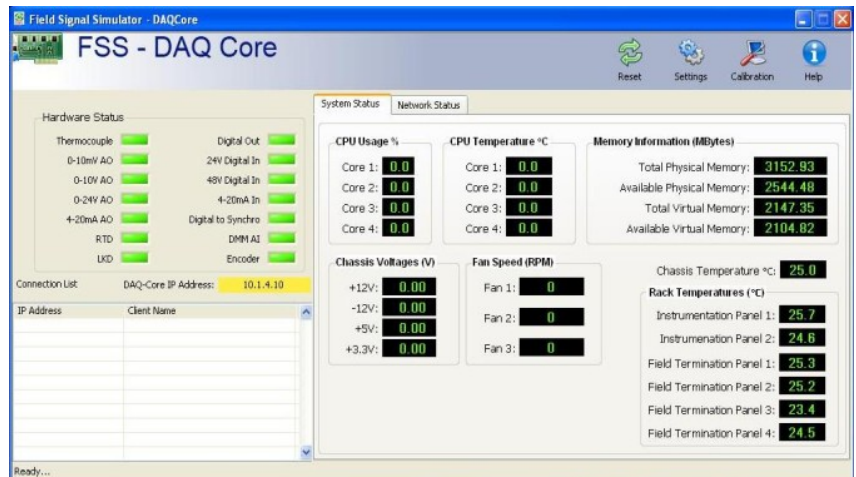

\section{FSS Server}

FIGURE 4: DAQ CORE - Snapshot for System Status for Server

First FSS server software is launched on the PXI-controller. Server should be launched before launching the client $\mathrm{s} / \mathrm{w}$ of the IPCs or on the PXI-Controller. Client displays an error message if it's launched first and then the client application exists. FSS Software runs on the background with its icon minimized to the windows taskbar icon tray. The Server application also displays the health parameters of the FSS systems as well as the clients that are connected.The Server module interacts with hardware of the field signal simulation system. Server module is responsible for reading and writing to all hardware I/Os. The maximum number of signals that FSS supports is given in the Table. 1 below.

\begin{tabular}{|c|c|c|c|c|}
\hline $\begin{array}{l}\text { St. } \\
\text { No }\end{array}$ & Signal Type & Signal Range & Signal char acteristics & $\begin{array}{l}\text { No. of } \\
\text { Ch. }\end{array}$ \\
\hline \multirow{5}{*}{1} & \multirow{5}{*}{$\begin{array}{l}\text { Analog Outputs } \\
\text { (Signal Generation) }\end{array}$} & $\begin{array}{c}\text { K-Type } \\
\text { Themocouple }\end{array}$ & $0.5^{\circ} \mathrm{C}$ resolution & 500 \\
\hline & & $0-10 \mathrm{mV}$ & $\begin{array}{c}14 \text { bits @ } 25 \mathrm{mV} \text { FSR } \\
\text { (equivalent to } 1.53 \mu \mathrm{V} \text { Step) }\end{array}$ & 16 \\
\hline & & $0-10 \mathrm{~V}$ & 14 bit resolution & 600 \\
\hline & & $0-24 \mathrm{~V}$ & 14 bit te solution & 20 \\
\hline & & $4.20 \mathrm{~mA}$ & 14 bit resolution & 75 \\
\hline 2 & $\begin{array}{l}\text { Digital Outputs } \\
\text { (Signal Excitation) }\end{array}$ & Drycontact & $24 \mathrm{~V}$, electro-mechanical & 650 \\
\hline 3 & Analog Input monitoring & $4.20 \mathrm{~mA}$ & 14 bit resolution & 10 \\
\hline 4 & Relay Input Monitoring & Drycontact & $24 \mathrm{~V}$, electro-mechanical & 350 \\
\hline 5 & $\begin{array}{l}\text { Open-collector } \\
\text { monitoring }\end{array}$ & Open-collector & $\left(\mathrm{V}_{\mathrm{CE}}\right)_{\Delta, \mathrm{T}}=1.5 \mathrm{~V}$ & 50 \\
\hline 6 & $\begin{array}{l}\text { RTD (Pt-100) sensor } \\
\text { simulation }\end{array}$ & $-200^{\circ} \mathrm{C}$ to $850^{\circ} \mathrm{C}$ & $\begin{array}{c}\Delta 1^{\circ} \mathrm{C} \\
\text { (equivalent to } 0.3 \Omega \text { Step) }\end{array}$ & 30 \\
\hline 7 & $\begin{array}{l}\text { Leak Detector (LKD) } \\
\text { signal simulation }\end{array}$ & $\begin{array}{l}\text { Resistance arm } \\
\text { Simulation }\end{array}$ & $0 \Omega, 470 \Omega, 570 \Omega, O$ pen & 350 \\
\hline
\end{tabular}

TABLE 1: Max. no. of Signals supports FSS system 


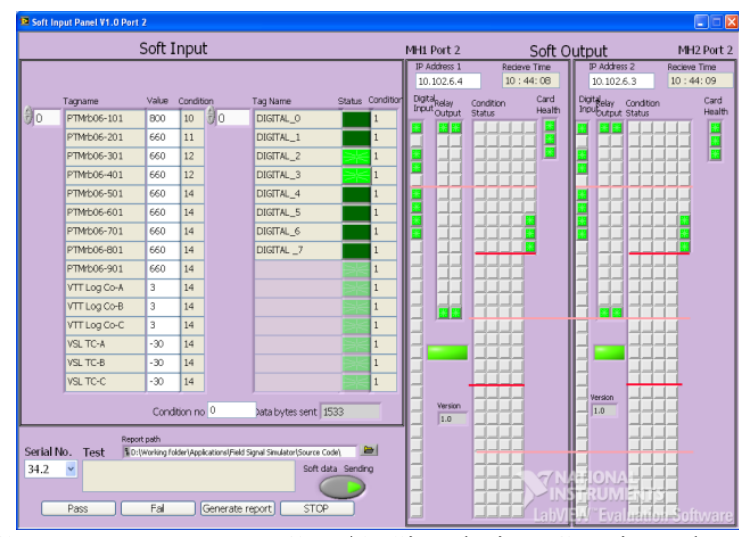

FIGURE 5: VME RTC - I/O Simulation GUI in LabVIEW

The Integration and System Testing process focuses on the functional externals, testing to uncover errors and to ensure that the defined input will produce actual results that agree with required results. All the cards designed on VME bus incorporate extensive online diagnostics features for fault detection and health monitoring through the watchdog timer. The watchdog timer forms the heartbeat of the entire computer system. Since plant outputs are controlled through actuation of relays, watchdog features were also integrated into the Mono-shot Relay Output board. This ensures that, in case of either system power failures or the failures of the ED20 CPU software, the plant outputs terminates to fail safe mode, thus preventing the catastrophe on the operating plant.

\section{Simulation And Experimental Results}

The software has to be installed in the PC, which is a onetime process. Double click on the setup.exe file of SYS_GUI folder. Continue through the steps to install the software as it being easy and self-explanatory. After installation, follow the below mentioned steps to execute the software.

i. $\quad$ Go to Start $\backslash$ Programs $\backslash S Y S$ GUI and run the application 'SYS_GUI'.

ii. A 'System Configuration' window opens as in the diagram below. The default settings are given in the Table 8.8 below.

\begin{tabular}{|c|c|}
\hline I/O Card & Default values \\
\hline Analog Input & 0 \\
\hline Digital Input & 0 \\
\hline Relay Output & 0 \\
\hline Analog Output & 0 \\
\hline Table 2: Default Settings I/O cards
\end{tabular}

Scan Interval and VME sub system number are to be selected.

In each scan, the CPU card scans all the inputs, updates the outputs and sends the data to GUI display station through Ethernet. The time gap between two successive scans is to be configured through the 'System Configuration' window.

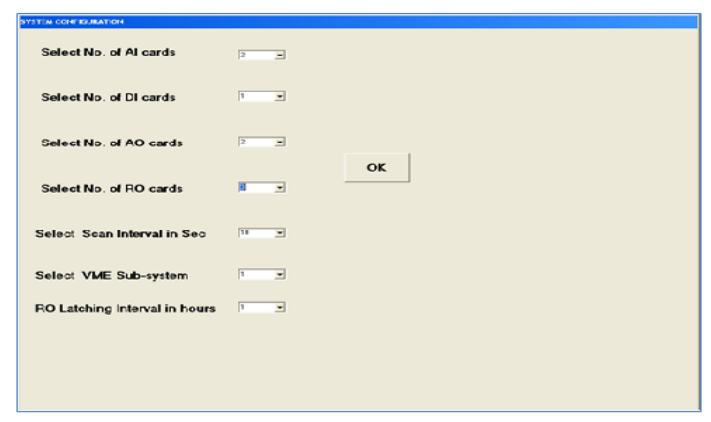

Safety Class 2 systems are configured with dual redundant VME subsystems with a Switch over Logic System (SOLS). To facilitate the testing of both the subsystems, the subsystem number has to be set in the 'System Configuration' window. The default values of Scan Interval, RO Latching Interval and VME sub system are given below After this configuration, when OK is pressed, these details are transferred to the VME subsystem and a new window 'Hardware Integration Test' along with the VME system number selected opens 
in GUI station. The Menu bar in this window has three options, Port 1, Port 2 and RO_Command. A screenshot is shown below.

Clicking on RO_Command will open a window asking for Card Number and selection among 2 Options- Single Channel Selection or Pattern selections. Once you click on Pattern Selection, a drop down list with 4 types of Patterns can be seen as in figure below. The output cards latches their outputs based on the pattern selected. Note that irrespective of the pattern selected, in next scan cycle, RO cards get latched to the next pattern in the sequence it was following after I/O card configuration.

Clicking on Single Channel Selection allows you to change any Channel of RO card ON/OFF as depicted in the following figure.

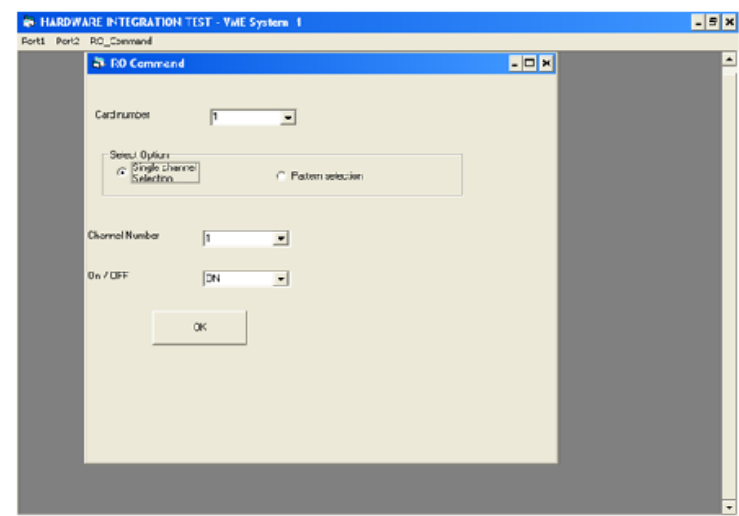

Click on Port 1 or port2 and a drop down list for 4 types of cards can be seen as in the figure below. Clicking on any of the selection (AI, DI, AO \& RO) will open a popup window with the name 'Hardware integration test-(Port 2-AI cards)' for AI, 'Hardware integration test-(Port 2-DI cards)' for DI, 'Hardware integration test-(Port 2-AO cards)' for $\mathrm{AO}$ and 'Hardware integration test-(Port 2-RO cards)' for RO. These windows display the information pertaining to respective type of card received from Ethernet Port2 of CPU card.

Screenshots of windows displaying the scanned data sent through port2 for all the cards (individually) is shown in the following figures.

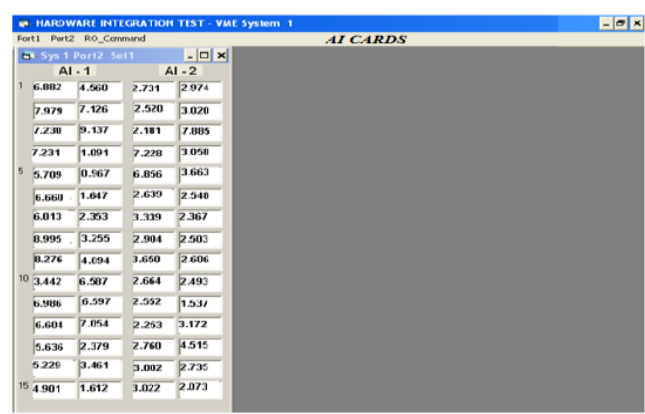

A screenshot of all the cards together is shown below. Similarly for port 1, clicking on the card's name in the drop down menu opens a popup window with the name 'Hardware integration test-(Port 1-AI cards)' for AI, 'Hardware integration test-(Port 1-DI cards)' for DI, 'Hardware integration test-(Port 1-AO cards)' for AO and 'Hardware integration test-(Port 1-RO cards)' for RO and displays the details coming through the port 1.

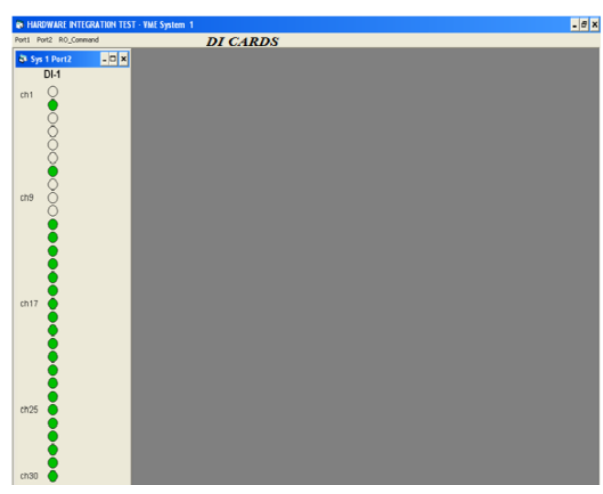



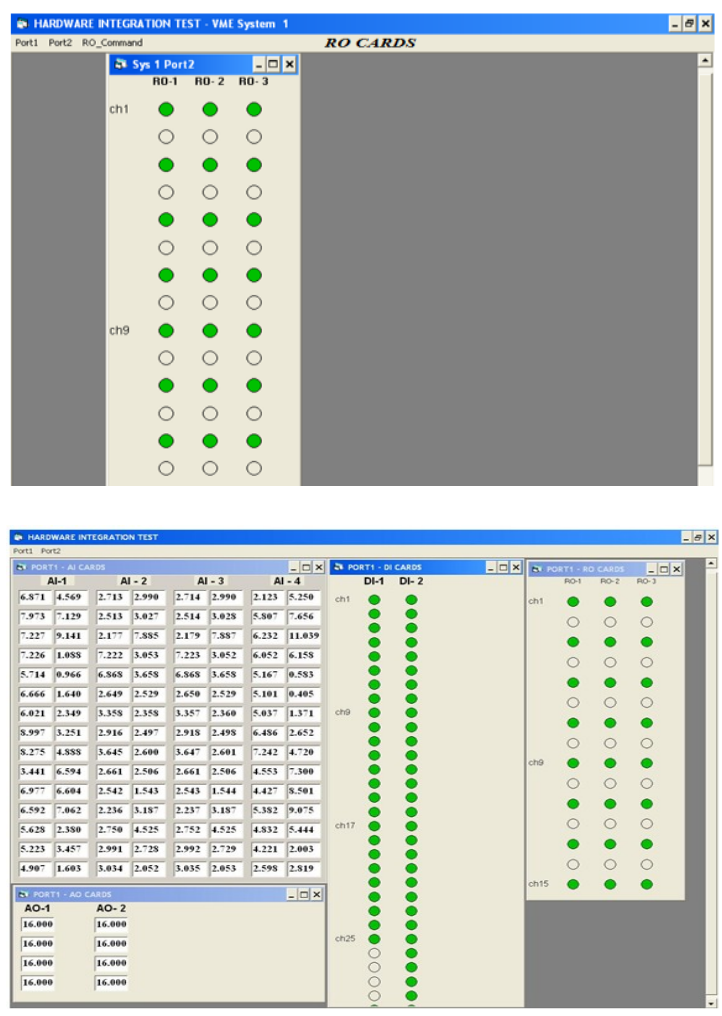

IV. Conclusion

FSS can generate Test Reports after successfully completion of system integration testing activity. FSS provides a method to test and verify the logic as per system requirements and generate reliable and accurate test results. More than 100 Real Time Computer based control systems for Power Plant Control \& Process Application have been developed and tested with Field Signal Simulator. Dynamic testing has ensured timing cycles and speeded up the testing time drastically. This method of testing can be adopted for any Microprocessor and Microcontroller based systems. This testing process has improved system reliability, flexibility and its performance. This FSS can be useful to develop and test ay control \& process systems which having a different I/O's needs dynamic simulation to reduce system failures and testing time and improve the functionality.

\section{References}

[1]. John AStankovic "RealTime Computing", University of Massachusett, April 161992

[2]. KANG G. SHIN AND PARAMESWARAN RAMANATHAN," Real-Time Computing: A New Discipline of Computer Science and Engineering" PROCEEDINGS OF THE IEEE, VOL. 82,NO. I , JANUARY 1994.

[3] ELEC 464 : MICROCOMPUTER SYSTEM DESIGN 1996/97 WINTER SESSION TERM 1" The System Bus" 